\title{
Tendencias y características de la mortalidad chilena 1970-2003
}

\author{
Ernesto Medina L y Ana Kaempffer R. \\ Trends and main features \\ of Chilean mortality
}

Background: Mortality data of a community allow to identify its severest diseases and health problems. Aim: To report epidemiologic data on Chilean mortality. Material and methods: Information about mortality was obtained from the World Health Organization, Pan American Health Organization, the Chilean Institute of Statistics and Ministry of Health. Results: A continuously declining trend in the risk of dying during the last 30 years was observed. Chile has the best situation among South American countries. Infant and general mortality rates fell in 89 and 39\%, respectively, during the period 1970-2003. This reduction is observed in all Chilean geographical regions. At the county level, there are 11 counties that duplicate the figure of mortality of the 337 other counties. In the case of infant mortality, there are 91 counties that duplicate the national figure, indicating an important inequity. Mortality is significantly associated with population aging, availability and use of hospital beds. There is a lower level of association with the proportion of population living in urban areas and poverty levels. A projection until 2010 shows and increasing mortality caused by malignant tumors and diabetes and a declining trend for circulatory, respiratory and digestive diseases and accidental deaths. Conclusions: Mortality has been managed efficiently in Chile but future trends underscore the need for cancer and diabetes prevention and management programs (Rev Méd Chile 2007; 135: 240-50).

(Key words: Diabetes mellitus; Life expectancy; Mortality; Neoplasms; Vital statistics)

Recibido el 14 de noviembre, 2005. Aceptado el 14 de julio, 2006.

Escuela de Salud Pública, Facultad de Medicina, Universidad de Chile

L a magnitud de la mortalidad en una comunidad se suele emplear como elemento indicador de la condición y nivel de salud de ella. El hecho se entiende por cuanto las muertes constituyen los problemas de salud más graves de una colectividad.

Su uso se facilita, ya que los países tienen información disponible, lo que a su vez depende de

Correspondencia a: Dr. Emesto Medina Lois. Independencia 939, Santiago. Fax: 7377121. E mail: emedina@med.uchile.cl la obligación existente de la certificación médica de las defunciones. En todo caso, no es fácil conseguir que la totalidad de las muertes sean registradas, especialmente en países de geografía difícil, o que la gran mayoría de los certificados de defunción sean extendidos por los médicos tratantes. Para tener un lenguaje común, los países utilizan la Clasificación Internacional de Enfermedades, la que es revisada y modificada cada 10 años; Chile, en el momento actual está empleando la $10^{\text {a }}$ Revisión.

En el país trabajan conjuntamente tres instituciones en la materia: el Registro Civil, que recibe 
los certificados de defunción y los almacena; el Instituto Nacional de Estadística (INE) y el Ministerio de Salud que elaboran la información.

Nos ha interesado explorar las tendencias de mortalidad chilenas, por cuanto el país ha mejorado su condición económica con aumento importante del PIB, ha modificado la organización de salud y, por otra parte, el ejercicio de la medicina se hace hoy día en forma diferente al pasado reciente. El estudio de las tendencias revela el éxito o fracaso de los programas de control de las diferentes patologías y la proyección de dichas tendencias apunta a las necesidades del futuro, más o menos inmediato.

\section{MateRIAL Y MÉTODO}

Para el estudio se han utilizado los datos de que dispone el Instituto Nacional de Estadística en cuanto a población y mortalidad ${ }^{1,2}$ y de la información del Ministerio de Salud ${ }^{3}$. Se han utilizado, igualmente, fuentes de información de la $\mathrm{OPS}^{4}$ y la $\mathrm{OMS}^{5}$. La modificación de la mortalidad según distintas variables, se ha concentrado en lo ocurrido en los últimos 30 años (1970-2003), por cuanto es el período que se vincula con las modificaciones antes mencionadas de la realidad chilena. Se han calculado las tasas de la mortalidad global, las variaciones según sexo y edad, las regiones, provincias y comunas y las patologías que generan defunciones. Para el estudio de asociaciones se ha empleado coeficientes de correlación de Spearman. La esperanza de vida chilena para el año 2000 ha sido calculada por nosotros. Para estimar la letalidad de las patologías, se combinan los datos de hospitalización $(\mathrm{H})$ y mortalidad (M) chilena en la fórmula M/H x 100.

\section{Resultados}

Magnitud de la mortalidad en el mundo. En la Tabla 1, se presentan indicadores de mortalidad de los países de Sudamérica, junto a los valores más altos y más bajos de la región de las Américas. Chile aparece con la mejor esperanza de vida al nacer, la menor mortalidad del primer año de vida, que llamamos mortalidad infantil, y la tercera ubicación en cuanto a mortalidad general de Sudamérica.
Tendencias de la mortalidad en Chile. De acuerdo a los datos chilenos (Tabla 2), la mortalidad general cayó en el siglo XX de 35,9 a 5,3 por mil. Este descenso de 30,6 puntos, corresponde a $86 \%$ de reducción. En la primera mitad del siglo descendió de 35,9 a 15,0 (20,9 puntos, $58 \%$ de reducción y entre 1950 y 2003 cayó en 9,8 puntos, 65\%). Al finalizar el siglo (1970-2000) bajó en 3,2 puntos (71\%). La tendencia al envejecimiento tiende a frenar el descenso de la mortalidad general. Cuando las tasas se ajustan por edad, usando como población estándar la chilena del año 2000, se aprecia que con dicho ajuste la mortalidad de 1970 habría sido 40\% mayor. El descenso de la mortalidad infantil es aún más marcado, disminuyendo de 342 por mil nacidos vivos en 1900, a 7,8 en el año 2000 (334 puntos, $95 \%)$.

Tendencias en las regiones chilenas. La Tabla 3 muestra la mortalidad general de las regiones en 1970 y 2003. En todas ellas hay reducción, que varía entre 4,2 y 1,1 puntos (17\% a $45 \%$ ). Las mayores caídas se observan en la VI, VII y VIII que, junto a la IX y X, eran las de mayor riesgo al comienzo del período, mientras los menores descensos ocurrieron en la XII región. En el año 2003 existía poca variación entre las regiones, fluctuando entre 6,1 en la V y 4,0 en la tercera. La mortalidad infantil sigue un patrón de disminución similar.

Tendencias de las causas de muerte. La Tabla 4 muestra la evolución de las principales causas de muerte en Chile en el período 1970-2003. Se advierte que los problemas infecciosos, nutricionales por déficit y neonatales han disminuido entre $86 \%$ y $100 \%$. Las enfermedades circulatorias descienden entre $15 \%$ y $23 \%$. Resulta preocupante advertir que el total de cánceres, y en particular la localización en la vesícula biliar y broncopulmonar han aumentado significativamente, situación que contrasta con el descenso proporcional de cáncer gástrico (48\%) y de cuello uterino (27\%). Se aprecia una tendencia al incremento de las patologías pulmonares vinculadas al consumo de tabaco, como la bronquitis crónica $(+58 \%$ ) y el cáncer broncopulmonar (+60\%). Hay una clara tendencia al alza de las defunciones por diabetes (+ 133\%) y por hipertensión arterial (+101\%). 
Tabla 1. M ortalidad en la región de las Américas. 2003

\begin{tabular}{|c|c|c|c|c|c|}
\hline \multicolumn{2}{|c|}{$\begin{array}{l}\text { La mayor expectativa } \\
\text { de vida }\end{array}$} & \multicolumn{2}{|c|}{$\begin{array}{l}\text { Menor mortalidad } \\
\text { general }\end{array}$} & \multicolumn{2}{|c|}{$\begin{array}{l}\text { Menor mortalidad } \\
\text { infantil }\end{array}$} \\
\hline Caimán & 79,2 & Costa Rica & 4,0 & Aruba & 1,5 \\
\hline Martinica & 79,1 & Belice & 4,3 & Caimán & 1,6 \\
\hline Canadá & 79,0 & I. Vírgenes & 4,4 & Bermuda & 3,0 \\
\hline \multicolumn{2}{|l|}{ Sudamérica } & \multicolumn{2}{|l|}{ Sudamérica } & \multicolumn{2}{|l|}{ Sudamérica } \\
\hline Chile & 75,6 & Venezuela & 4,7 & Chile & 10 \\
\hline Uruguay & 75,0 & Paraguay & 5,1 & Uruguay & 14 \\
\hline Argentina & 73,8 & Chile & 5,7 & Argentina & 17 \\
\hline Venezuela & 73,0 & Ecuador & 5,8 & Paraguay & 19 \\
\hline Colombia & 71,9 & Perú & 6,2 & Venezuela & 19 \\
\hline Ecuador & 71,3 & Colombia & 6,4 & Colombia & 20 \\
\hline Paraguay & 70,4 & Brasil & 7,0 & Ecuador & 30 \\
\hline Perú & 69,5 & Argentina & 7,8 & Brasil & 32 \\
\hline Brasil & 68,3 & Bolivia & 8,2 & Perú & 33 \\
\hline Bolivia & 63,5 & Uruguay & 9,3 & Bolivia & 67 \\
\hline \multicolumn{2}{|c|}{ Peor expectativa } & \multicolumn{2}{|c|}{$\begin{array}{l}\text { Peor mortalidad } \\
\text { general }\end{array}$} & \multicolumn{2}{|c|}{$\begin{array}{l}\text { Peor mortalidad } \\
\text { infantil }\end{array}$} \\
\hline Bolivia & 62,0 & Uruguay & 9,3 & Guatemala & 37 \\
\hline Guyana & 62,4 & Guyana & 9,4 & Honduras & 42 \\
\hline Haití & 53,3 & Haití & 12,6 & Haití & 80 \\
\hline
\end{tabular}

(Fuente: Datos oficiales de OMS y OPS.).

Tabla 2. M ortalidad general, mortalidad infantil y esperanza de vida al nacer. C hile 1900-2003

\begin{tabular}{|c|c|c|c|c|c|c|}
\hline \multirow[t]{2}{*}{ Año } & \multicolumn{2}{|c|}{ Mortalidad general } & \multicolumn{2}{|c|}{ Mortalidad infantil } & \multicolumn{2}{|c|}{ Esperanza de vida } \\
\hline & $\begin{array}{c}\text { Tasa por } \\
1.000\end{array}$ & $\begin{array}{c}\text { \% variación } \\
\text { anual }\end{array}$ & $\begin{array}{c}\text { Tasa por } \\
1.000\end{array}$ & $\begin{array}{c}\text { \% variación } \\
\text { anual }\end{array}$ & Años & $\%$ aumento \\
\hline 1900 & 35,9 & & 342 & & & \\
\hline 1910 & 38,8 & $+0,8$ & 267 & $-2,19$ & & \\
\hline 1920 & 30,7 & $-2,1$ & 263 & $-0,11$ & 31 & \\
\hline 1930 & 24,5 & $-2,0$ & 234 & $-1,10$ & 40 & 2,9 \\
\hline 1940 & 21,5 & $-1,2$ & 217 & $-0,73$ & 42 & 2,2 \\
\hline 1950 & 15,0 & $-3,0$ & 136 & $-3,73$ & 55 & 3,1 \\
\hline 1960 & 12,4 & $-1,7$ & 120 & $-1,18$ & 57 & 1,8 \\
\hline 1970 & 8,5 & $-3,1$ & 79 & $-3,12$ & 62 & 0,9 \\
\hline 1980 & 6,6 & $-2,2$ & 33 & $-5,12$ & 71 & 1,5 \\
\hline 1990 & 6,0 & $-1,0$ & 16 & $-5,15$ & 74 & 1,1 \\
\hline 2000 & 5,2 & $-1,3$ & 7,8 & $-5,45$ & 75 & 0,7 \\
\hline 2003 & 5,3 & & & & & \\
\hline
\end{tabular}

Fuente: Datos básicos Instituto Nacional de Estadística. Anuarios de Demografía. Cálculos de los autores. 
Tabla 3. M ortalidad general e infantil en las regiones chilenas. 1970 y 2003

\begin{tabular}{|lrccrrr|}
\hline Regiones & \multicolumn{3}{c}{$\begin{array}{c}\text { Mortalidad general } \\
\text { Por 1.000 habitantes }\end{array}$} & \multicolumn{3}{c|}{ Mortalidad infantil } \\
& 1970 & 2003 & $\%$ disminución & 1970 & 2003 & $\%$ disminución \\
\hline Chile & 8,5 & 5,3 & 38 & 80 & 7,8 & 89 \\
I & 7,7 & 4,7 & 30 & 59 & 8 & 86 \\
II & 7,8 & 4,9 & 30 & 74 & 9 & 88 \\
III & 7,2 & 4,0 & 44 & 96 & 8 & 92 \\
IV & 7,8 & 5,0 & 36 & 97 & 9 & 91 \\
V & 6,4 & 6,1 & 27 & 83 & 8 & 90 \\
Met & 7,6 & 4,9 & 36 & 58 & 8 & 86 \\
VI & 8,9 & 5,4 & 37 & 89 & 10 & 89 \\
VII & 10,4 & 5,8 & 44 & 116 & 8 & 93 \\
VIII & 9,5 & 5,4 & 43 & 110 & 10 & 91 \\
IX & 9,8 & 6,0 & 39 & 150 & 10 & 94 \\
X & 9,8 & 6,9 & 39 & 141 & 8 & 94 \\
XI & 6,8 & 4,3 & 37 & 86 & 10 & 88 \\
XII & 7,1 & 6,0 & 15 & 38 & 9 & 76 \\
\hline
\end{tabular}

Fuente: INE Chile. Demografía 1970 y Estadísticas Vitales 2003.

Tabla 4. Evolución de las principales causas de muerte en Chile 1970 y 2003. Tasas por 100.000

\begin{tabular}{|lrrrr|}
\hline Causas específicas & \multicolumn{2}{c}{ Tasas por 100.000 } & \multicolumn{2}{c|}{$\%$ variación } \\
& 1970 & 2003 & Total & Anual \\
\hline Sarampión & 70 & 0 & -100 & $-3,0$ \\
Diarreas & 43 & 1,4 & 97 & $-2,9$ \\
Tuberculosis & 25 & 1,8 & 93 & $-2,8$ \\
Perinatales* & 44 & 4,8 & 89 & $-2,0$ \\
Neumonías & 132 & 27 & 86 & $-2,6$ \\
Septicemias & 8 & 0,9 & 85 & $-2,6$ \\
Desnutrición & 16 & 3,2 & 80 & $-2,4$ \\
Colecistopatías & 7 & 1,6 & 77 & $-2,3$ \\
Cardiopatía reumática & 6 & 1,7 & 70 & $-2,1$ \\
Cáncer gástrico & 34 & 20 & 48 & $-0,6$ \\
Congénitas & 10 & 5,8 & 39 & $-1,2$ \\
Cáncer cuello uterino & 12 & 8,6 & 27 & $-0,8$ \\
Cirrosis hepática & 31 & 23 & 26 & $-0,8$ \\
TEC & 19 & 15 & 21 & $-0,6$ \\
Cardiopatía coronaria & 62 & 52 & 16 & $-0,5$ \\
AVE & 60 & 51 & 15 & $-0,0$ \\
Cáncer vesícula biliar & 9,5 & 12,3 & +29 & $+0,8$ \\
Bronquitis crónica & 10 & 16 & +58 & $+1,8$ \\
Cáncer pulmonar & 8 & 12,8 & +60 & $+2,8$ \\
Hipertensión arterial & 8,5 & 17,1 & +101 & $+4,0$ \\
Diabetes mellitus & 9 & 21,0 & +133 & \\
\hline
\end{tabular}

*Representa un grupo de casos específicos.

Fuente: INE Chile. Demografía 1970 y Estadísticas Vitales 2003. Las causas de muerte están ordenadas de acuerdo al porcentaje de variación total del periodo. 
La mortalidad general del país bajó desde 883 por 100.000 habitantes en 1970 a 528 en 2003. Esta disminución se debió especialmente a la caída de las muertes por enfermedades infecciosas (84 por 100.000), circulatorias (68), diarreas infantiles (65), muerte neonatal (59), accidentes y violencias (34) y tuberculosis (36). También disminuyeron en proporciones menores otras enfermedades infecciosas y cirrosis hepática (12 por 100.000).

Mortalidad según sexo y edades. Los datos de la Tabla 5 revelan que el descenso de mortalidad entre 1970 y 2003 fue similar en ambos sexos (39\%). Se aprecia que la caída del riesgo se registró en todos los grupos de edad. El mayor descenso, de $90 \%$, correspondió a la mortalidad de menores de 10 años.

Variaciones mensuales. Entre 1970 y 2003 se mantuvo la situación clásica en el país de exceso de la mortalidad general entre junio y septiembre (Tabla 6). En cambio, en la mortalidad infantil, dicho aumento se observa en los meses cálidos entre diciembre y febrero.

Tabla 5. M ortalidad según sexo y edad, C hile 1970 y 2003. Tasas por 100.000 habitantes

\begin{tabular}{|lrrrrrrrrr|}
\hline Edad & \multicolumn{3}{c}{ Hombres } & \multicolumn{3}{c}{ Mujeres } & \multicolumn{3}{c|}{ Total } \\
& 1970 & 2003 & $\begin{array}{c}\text { Variación } \\
\%\end{array}$ & 1970 & 2003 & $\begin{array}{c}\text { Variación } \\
\%\end{array}$ & 1970 & 2003 & $\begin{array}{c}\text { Variación } \\
\%\end{array}$ \\
\hline-10 & 1.153 & 90 & 92 & 972 & 82 & 92 & 1.081 & 87 & 92 \\
$10-19$ & 116 & 46 & 60 & 78 & 19 & 76 & 87 & 33 & 62 \\
$20-29$ & 255 & 131 & 49 & 151 & 36 & 76 & 215 & 84 & 61 \\
$30-39$ & 422 & 182 & 57 & 259 & 65 & 75 & 339 & 123 & 64 \\
$40-49$ & 829 & 319 & 62 & 462 & 165 & 64 & 636 & 241 & 62 \\
$50-59$ & 1.648 & 761 & 54 & 927 & 421 & 55 & 1.345 & 586 & 55 \\
$60-69$ & 3.722 & 1.892 & 49 & 2.461 & 1.057 & 57 & 2.938 & 1.445 & 51 \\
$70-79$ & 7.329 & 5.087 & 31 & 5.477 & 2.889 & 47 & 6.292 & 3.781 & 40 \\
$80+$ & 11.080 & 7.266 & 30 & 12.953 & 7.251 & 14 & 12.123 & 7.466 & 38 \\
\hline
\end{tabular}

Fuente: INE Chile. Demografía 1970 y Estadísticas Vitales 2003. Càlculos de los autores.

Tabla 6. Variación estacional de los más frecuentes grupos de causas de muerte. C hile. 2000

\begin{tabular}{|lcccccc|}
\hline Mes & Circulatorias & Respiratorias & Cánceres & Accidentes & Otras & Total \\
\hline I & 1.845 & 532 & 1.602 & 714 & 2.043 & 6.736 \\
II & 1.505 & 443 & 1.471 & 605 & 1.755 & 5.779 \\
III & 1.668 & 461 & 1.651 & 590 & 1.927 & 6.297 \\
IV & 1.824 & 567 & 1.633 & 552 & 1.940 & 6.516 \\
V & 2.193 & 690 & 1.648 & 636 & 2.238 & 7.405 \\
VI & 2.174 & 657 & 1.591 & 570 & 2.163 & 7.055 \\
VII & 2.393 & 898 & 1.780 & 645 & 2.540 & 8.246 \\
VIII & 2.511 & 912 & 1.730 & 595 & 2.491 & 8.239 \\
IX & 2.024 & 676 & 1.658 & 619 & 2.186 & 7.163 \\
X & 2.025 & 572 & 1.677 & 639 & 2.039 & 6.942 \\
XI & 1.840 & 522 & 1.617 & 637 & 1.961 & 6.577 \\
XII & 1.789 & 502 & 1.642 & 605 & 2.079 & 6.617 \\
Total & 23.771 & 7.432 & 19.700 & 7.407 & 25.362 & 83.672 \\
\hline
\end{tabular}

Fuente: INE. Estadísticas Vitales 2000. Cálculos del autor. 
Seguimiento de cohortes según año de nacimiento. La Tabla 7 muestra que las generaciones nacidas en los últimos años en Chile, a lo largo de la existencia, han tenido menores riesgos de muerte que los nacidos en la primera mitad del siglo XX.

Factores asociados a la mortalidad chilena. En la Tabla 8 se muestra la magnitud de asociación con diversos factores, de la mortalidad general e infantil de las regiones chilenas, estudiada por coeficientes de Spearman. La asociación es muy alta y significativa para el porcentaje de vejez de la población y los egresos hospitalarios. Aparecen con menor asociación y menor significación la proporción de población urbana y de pobreza de las regiones.

Calidad de la información. En los últimos 40 años se ha logrado reducir a menos de la mitad el porcentaje de muertes por causas mal definidas y aumentar la certificación médica de las defunciones a $98,5 \%$ de ellas. En cuanto al origen de la certificación actual, la mitad de las muertes ocurren en servicios hospitalarios, una proporción similar en los domicilios y cerca de $10 \%$, calificada como otra, corresponde a autopsias médicolegales.

En las Tablas 9 y 10 aparecen los valores de mortalidad general e infantil de las comunas chilenas. Cifras muy bajas de mortalidad pueden observarse en localidades muy pequeñas y en comunas en las que sus habitantes fallecen en otras comunas en las que han solicitado atención médica. Conviene señalar que las muertes pueden adjudicarse a las comunas en que se producen y registran decesos -ocurrencia- 0 al sitio en que vivían los fallecidos, o residencia. A nivel regional, se advierte que las tasas de mortalidad calculadas por residencia u ocurrencia son similares. Algo parecido ocurre con los datos de provincias. A diferencia de ello, a nivel de las comunas se aprecia que algunas de ellas atraen pacientes graves y la tasa de ocurrencia supera netamente a la de residencia. Ello ocurre en las comunas que

Tabla 7. Riesgo de muerte en cohortes chilenas según año de nacimiento

\begin{tabular}{|c|c|c|c|c|c|c|c|c|}
\hline \multirow{2}{*}{$\begin{array}{l}\text { Tasas } \\
\text { a la edad }\end{array}$} & \multicolumn{8}{|c|}{ Año de nacimiento. Tasas por 1.000} \\
\hline & 1920 & 1930 & 1940 & 1950 & 1960 & 1970 & 1980 & 1990 \\
\hline $0-4$ & 419 & 316 & 317 & 181 & 89 & 98 & 38 & 19 \\
\hline $5-9$ & 36 & 26 & 20 & 10 & 5 & 4 & 2 & 1 \\
\hline $10-14$ & 23 & 23 & 12 & 5 & 3 & 3 & 2 & \\
\hline $15-19$ & 43 & 31 & 13 & 5 & 5 & 4 & 1 & \\
\hline $20-24$ & 63 & 27 & 15 & 9 & 6 & 6 & & \\
\hline $25-29$ & 53 & 18 & 17 & 9 & 6 & 6 & & \\
\hline $30-34$ & 39 & 17 & 17 & 9 & 7 & 6 & & \\
\hline 35-39 & 38 & 25 & 18 & 10 & 8 & & & \\
\hline $40-44$ & 44 & 27 & 22 & 15 & 8 & & & \\
\hline $45-49$ & 50 & 32 & 29 & 15 & & & & \\
\hline $50-54$ & 69 & 44 & 40 & 23 & & & & \\
\hline $55-59$ & 79 & 57 & 51 & & & & & \\
\hline $60-64$ & 117 & 81 & 68 & & & & & \\
\hline $65-69$ & 174 & 111 & & & & & & \\
\hline $70-74$ & 279 & 151 & & & & & & \\
\hline $75-79$ & 406 & & & & & & & \\
\hline $80+$ & 841 & & & & & & & \\
\hline
\end{tabular}

Fuente: Datos básicos del INE. Cálculos del autor. 
Tabla 8. Correlación de la mortalidad general e infantil en las regiones chilenas con factores determinantes. Chile 2003. C oeficiente de Spearman

\begin{tabular}{|lc|}
\hline Mortalidad general & Coeficientes \\
\hline Estructura étnica & 0,02 \\
Porcentaje desnutrición infantil & 0,05 \\
Recursos médicos & 0,07 \\
Atenciones ambulatorias por persona & 0,10 \\
Malnutrición por exceso & 0,23 \\
Desocupación & 0,25 \\
Escolaridad de la población & 0,25 \\
Alfabetismo de la población & 0,27 \\
Porcentaje de pobreza & $0,40^{*}$ \\
Porcentaje de residencia urbana & $0,48^{*}$ \\
Disponibilidad de camas hospitalarias & $0,58^{* *}$ \\
Egresos hospitalarios por 1.000 personas & $0,76^{* *}$ \\
Envejecimiento poblacional & $0,81^{* *}$ \\
Mortalidad infantil & \\
Porcentaje atención profesional del parto & \\
Desocupación & 0,01 \\
Estructura étnica & 0,01 \\
Atenciones médicas ambulatorias por persona & 0,09 \\
Porcentaje de residencia urbana & 0,10 \\
Porcentaje de pobreza & 0,13 \\
Egresos hospitalarios por persona & 0,17 \\
Alfabetismo de la población & 0,21 \\
Camas pediátricas & 0,22 \\
Envejecimiento poblacional & $0,47^{*}$ \\
\hline
\end{tabular}

Fuente: Datos básicos del INE y el Ministerio de Salud. Cálculos de los autores.

$*_{p}<0,05 .{ }^{*} p<0,01$.

Tabla 9. M ortalidad general en las comunas de Chile

\begin{tabular}{|lcrrccrr|}
\hline Región & $\begin{array}{c}\text { Tasa } \\
\text { regional }\end{array}$ & \multicolumn{6}{c|}{ Número de comunas según tasa de mortalidad general } \\
& & 0 a 4 & 5 a 9 & 10 a 14 & 15 a 24 & $25+$ & Total \\
\hline Chile & 5,3 & 104 & 23 & 6 & 3 & 2 & 246 \\
I & 4,7 & 11 & 2 & - & - & - & 13 \\
II & 4,9 & 5 & 3 & - & - & - & 9 \\
III & 4,0 & 7 & 2 & - & - & - & 9 \\
IV & 5,0 & 4 & 11 & - & - & - & 15 \\
V & 8,1 & 12 & 26 & 1 & - & - & 39 \\
Metr & 4,9 & 27 & 25 & 1 & - & - & 53 \\
VI & 5,6 & 5 & 25 & 1 & - & - & 31 \\
VII & 5,8 & 5 & 24 & 1 & - & - & 30 \\
VIII & 5,4 & 7 & 45 & - & - & - & 52 \\
IX & 6,0 & 4 & 28 & - & - & - & 32 \\
X & 6,0 & 7 & 33 & 2 & - & - & 42 \\
XI & 4,3 & 4 & 5 & - & - & 1 & 10 \\
XII & 6,0 & 6 & 3 & - & 2 & - & 11 \\
\hline
\end{tabular}

Fuente: Datos básicos del INE. Cálculos de los autores. 
Tabla 10. M ortalidad infantil en las comunas chilenas. Año 2003

\begin{tabular}{|lrrrrrrr|}
\hline Región & Tasa & \multicolumn{7}{c|}{ № de comunas según tasa de mortalidad infantil } \\
& regional & $0-8$ & $9-16$ & por 1.000 nacidos vivos & \\
& & 94 & 110 & 76 & 37 & 26 & 343 \\
\hline Chile & 7,8 & 9 & 2 & - & - & - & 11 \\
I & 8,8 & 5 & 2 & - & 1 & 1 & 9 \\
II & 9,4 & 1 & 3 & 1 & 2 & 2 & 9 \\
III & 7,2 & 5 & 2 & 4 & 2 & 2 & 15 \\
IV & 9,1 & 11 & 14 & 7 & 5 & 2 & 39 \\
V & 8,4 & 9 & 31 & 9 & 3 & - & 52 \\
Metr & 7,5 & 5 & 7 & 9 & 7 & 6 & 33 \\
VI & 10,3 & 3 & 15 & 7 & 2 & 2 & 29 \\
VII & 8,5 & 14 & 17 & 5 & 3 & 52 \\
VIII & 10,4 & 13 & 7 & 10 & 6 & 2 & 31 \\
IX & 10,5 & 11 & 10 & 4 & 3 & 41 \\
X & 8,4 & 13 & 11 & 1 & - & 3 & 10 \\
XI & 10,3 & 5 & 1 & 1 & - & 1 & 12 \\
XII & 8,5 & 9 & 1 & 1 & \\
\hline
\end{tabular}

Tabla 11. Riesgos de muerte en población general de C hile expresado en tasas específicas de causas de muerte por 100.000 habitantes. Proyección según tasas observadas en 1990 y 2003

\begin{tabular}{|c|c|c|c|c|c|}
\hline \multirow{2}{*}{\multicolumn{2}{|c|}{ Causas de muerte }} & \multicolumn{2}{|c|}{$\begin{array}{l}\text { Proyección de tasas } \\
\text { para el año } 2003\end{array}$} & \multicolumn{2}{|c|}{$\begin{array}{l}\text { Tasas observadas } \\
\text { por } 100.000\end{array}$} \\
\hline & & Tasas & $\%$ & 1990 & 2003 \\
\hline \multicolumn{2}{|c|}{ Total } & 484 & 100 & 600 & 530 \\
\hline & Circulatorias & 144 & 28,9 & 165 & 151 \\
\hline 2. & Cánceres & 134 & 26,8 & 108 & 125 \\
\hline 3. & Digestivas & 39 & 7,8 & 47 & 42 \\
\hline 4. & Traumatismos & 33 & 6,6 & 73 & 47 \\
\hline 5. & Respiratorias & 32 & 6,4 & 74 & 47 \\
\hline & Endocrino metabólicas & 32 & 6,4 & 14 & 26 \\
\hline 7. & Mentales & 21 & 4,2 & 9 & 17 \\
\hline 8. & Genitourinarias & 15 & 3,0 & 12 & 14 \\
\hline & Transmisibles & 9 & 1,8 & 19 & 12 \\
\hline 10. & Tumores benignos & 6 & 1,2 & 3,5 & 1,2 \\
\hline 11. & Mal definidas & 6 & 1,2 & 37 & 15 \\
\hline & Aparato locomotor & 3,4 & 0,7 & 2,0 & 2,9 \\
\hline & Congénitas & 3,3 & 0,7 & 10,4 & 5,8 \\
\hline 14. & Piel & 3,1 & 0,6 & 1,2 & 2,5 \\
\hline 15. & Sangre & 1,8 & 0,4 & 1,7 & 1,8 \\
\hline & Perinatales & 1,4 & 0,3 & 13,0 & 4,8 \\
\hline & Gestacionales & 0,1 & 0,0 & 0,90 & 0,19 \\
\hline
\end{tabular}

Las causas de muerte aparecen ordenadas según el volumen observado en el año 2003. 
son capitales de regiones y en las que tienen mayor desarrollo socioeconómico. En estas comunas, en el año 2000, ocurrieron 15.993 muertes de las que 11.831 eran residentes (índice de exceso de 1,35). A título de ejemplo, la diferencia entre ocurrencia y residencia es muy grande en la provincia de Iquique, en las que ocurrieron 19 fallecimientos en circunstancias que los fallecidos residentes fueron 54 (índice 2,84). Los hospitales de la ciudad de Santiago generan importantes diferencias para las comunas en las que se ubican. La diferencia fue muy alta para la comuna de Independencia con 5.872 ocurrencias y 797 muertes de residentes (índice 7,36), Providencia con 2.793 y 1.019 (índice 2,74) San Miguel con 1.664 y 684 (índice 2,4) o Santiago con 3.500 y 1.450 (índice 2,44).

Proyecciones para el año 2010. Si la tendencia del período 1990-2003 se proyecta hasta el año 2010, en Chile deberíamos esperar una mortalidad anual de 484 por 100.000 habitantes (Tabla 11). Se mantendrían las causas circulatorias, seguidas de cerca por los cánceres, como causas más frecuentes. Las enfermedades digestivas subirían al tercer lugar y aumentaría la importancia relativa de las muertes de origen endocrino metabólico, en particular la diabetes mellitus.

\section{Discusión}

En la misma forma que un objetivo fundamental de la medicina es salvar la vida del paciente, en el terreno colectivo, una meta importante es la de disminuir el riesgo de muerte de la comunidad ${ }^{6}$. Chile se ubica en el 15o lugar de los países del mundo en cuanto a mortalidad general y en el $17^{\circ}$ si consideramos la esperanza de vida al nacer. $\mathrm{Si}$ nos limitamos a Sudamérica, el país posee la mejor esperanza de vida (75,6 años) y la más baja mortalidad infantil (7,8 por mil). Si nos comparamos con Canadá, país desarrollado con buenos indicadores de salud, Chile tiene una esperanza de vida 3,4 años menor y registra el doble de mortalidad infantil.

Conviene destacar que el envejecimiento poblacional frena la disminución del riesgo de morir por la mayor probabilidad de defunción de las personas envejecidas. Desde 1910 disminuyó la mortalidad general de 38,8 a 5,3 por 1.000 . Sin embargo, en las dos últimas décadas del siglo XX, el porcentaje de reducción anual ha bajado significativamente, mientras en la mortalidad infantil, el porcentaje de reducción fue máximo entre 1970 y 2003.

La medicina chilena ha sido muy exitosa en los últimos 30 años para disminuir el riesgo de muerte por enfermedades infecciosas ${ }^{7}$ y por desnutrición. Ha habido progresos significativos en varias afecciones circulatorias ${ }^{8-10}$, traumatismos ${ }^{11}$, enfermedades respiratorias ${ }^{11}$ y hepatobiliares ${ }^{12-14}$. La contraparte es el aumento del riesgo del conjunto de los cánceres ${ }^{15}$, con la excepción del cérvico uterino ${ }^{16}$ y gástrico ${ }^{17}$. Las enfermedades asociadas al consumo de tabaco aumentan en el país, como igualmente las muertes debidas a hipertensión arterial y diabetes mellitus.

Todos los grupos etáreos de la población, y en forma especial los menores de 10 años, han disminuido su riesgo de muerte ${ }^{18-20}$. Una forma interesante de examinar la situación, la constituye el seguimiento y comparación de cohortes definidas según año de nacimiento, que revela un gran riesgo a lo largo de toda su existencia de los nacidos en 1920-1930, que contrasta con los menores valores de los nacidos en períodos recientes.

Las muertes de los chilenos varían a lo largo del año ${ }^{21}$, apareciendo un exceso entre junio y septiembre, el que se registra nítidamente en las muertes de origen circulatorio y respiratorio.

El estudio de asociación de diversos factores con la mortalidad de las regiones chilenas ha destacado el significado de variables de tipo económico ${ }^{22}$. Nosotros hemos encontrado que son factores asociados significativamente al riesgo el grado de envejecimiento poblacional, la dotación de camas hospitalarias y de egresos, y alcanzan valores límites, la proporción de población urbana y la pobreza. La asociación de factores es muy diferente para la mortalidad general y la infantil.

La calidad de la certificación de defunción puede medirse por las proporciones extendidas por médicos y las causas de muerte mal definidas. En Chile ha habido un gran mejoramiento global, particularmente en zonas urbanas, con sólo $0,6 \%$ de certificación por testigos en años recientes (6,5\% en zonas rurales). La proporción de testigos 
es alta en la $\mathrm{X}$ y IX regiones (4,8\% y $8,4 \%)$. Conviene destacar, sin embargo, que en la certificación del año 2003 sólo 34,4\% fue hecho por médicos tratantes y 13,9\% por legistas; $50,6 \%$ corresponde a médicos no tratantes y $1,1 \%$ a testigos.

Las tasas de mortalidad general oscilan en las comunas de Chile entre 0 y 83, esta última en Porvenir, con 4 muertes. De 21 comunas con valores muy bajos ( 0 a 2 por 1.000), 16 pertenecen a los extremos del país (I, II, XI y XII) y corresponden a áreas de escasa población. Sólo $3 \%$ de las comunas duplican la cifra nacional $(5,2$ por 1.000); 316 comunas tienen entre 3 y 17 por $1.000^{23}$. En el caso de la mortalidad infantil la situación es diferente: 91 comunas duplican el valor nacional y 65 de ellas se encuentran entre la VI y X regiones. El que una cuarta parte de las comunas tengan riesgos altos o muy altos es un hecho preocupante 24 .

Las cifras de letalidad chilenas revelan el alto riesgo de las enfermedades cardiovasculares, cánceres y enfermedades endocrino metabólicas, en particular la diabetes. El mínimo riesgo se observa en los problemas gestacionales y las enfermedades del aparato locomotor.

\section{REFERENCIAS}

1. INE. Anuarios de Estadísticas Vitales 1999-2003.

2. INE. Demografía 1920-1998.

3. Ministerio de Salud de Chile. Indicadores básicos de salud 2002 y 2003.

4. OPS. Situación de salud en las Américas. Publicación Científica № 587, Washington, EE.UU., 2002.

5. OMS World Health Statistics. 1996, Geneva, Switzerland.

6. Medina E. Expectativas de vida actuales de la población chilena. Rev Méd Chile 1999; 127 : 637-8.

7. Cabello F, SpRInger AD. Fiebre tifoidea en Chile 1977-1990: enfermedad emergente. Rev Méd Chile 1997; 125: 474-82.

8. Medina E, KaEMPFFer AM. La tendencia declinante de la mortalidad cardiovascular. Rev Chil Salud Pública 2001; 5: 63-8.
La proyección de los riesgos de muerte actuales hasta el año 2010 revela, en primer lugar, que la mortalidad chilena debería ser muy baja, ubicándose junto a Costa Rica como una de las dos menores del continente. Enfrentaremos una situación en la que las enfermedades circulatorias y los cánceres seguirán constituyendo las primeras causas de muerte y las enfermedades digestivas ${ }^{25}$ se ubicarán en el tercer lugar. Se mantendrán las patologías respiratorias, los traumatismos y las afecciones endocrino metabólicas en las seis primeras ubicaciones. Por otra parte, continuaría la disminución de las muertes infantiles y de las enfermedades infecciosas transmisibles.

El conocimiento de la situación actual y de sus proyecciones deberían tomarse muy en cuenta para la planificación de actividades en salud ${ }^{26}$. Parece urgente reforzar los esfuerzos de diagnóstico precoz de cánceres frecuentes, de la diabetes y la hipertensión arterial, y de tratamiento eficaz de neumonía y traumatismos. La información disponible apunta a que esos esfuerzos son los más correctos y necesarios en el control de la mortalidad chilena sin descuidar la investigación de factores de riesgo y distintos cambios de inadecuados estilos de vida.

9. Szот J. Mortalidad por infarto agudo al miocardio en Chile 1990-2001. Rev Méd Chile 2004; 132: 1227-34.

10. Castro P, Vukasovic JL, Garcés E. Insuficiencia cardiaca en hospitales chilenos Grupo ICARO. Rev Méd Chile 2004; 132: 655-62.

11. Medina E, KaempfFer AM, Cornejo E, Hernández E. El problema de los accidentes en Santiago. Cuad Med Soc 1994; 122: 1087-94.

12. Csendes A, Korn O, Medina E, Becerra M, Csendes P. Mortalidad de la cirugía biliar en Chile en 1990. Rev Méd Chile 1993; 121: 937-42.

13. Csendes A, Becerra M, Smok G, Medina E. Prevalencia del cáncer de la vesícula biliar en colecistectomías. Rev Méd Chile 1991; 119: 887-90.

14. Serra I, Calvo A, Maturana M, Medina E. Changing trends of gallbladder cancer in Chile. Int J Cancer 1990; 45: 376-7. 
15. Medina E, KaEMPFFer AM. Mortalidad por cáncer en Chile. Rev Méd Chile 2001; 129: 1195-202.

16. Guzmán S, Salas P, Puente R. Pesquisa y control del cáncer cérvico uterino en Valdivia. Rev Méd Chile 2005; 133: 685-92.

17. Csendes A, Smok G, Medina E, Salgado I, Rivera R, Quitral M. Características evolutivas del cáncer gástrico. Rev Méd Chile 1992; 120: 36-40.

18. KaEMPFFer AM, MEdina E. Análisis de la mortalidad infantil y factores condicionantes. Rev Chil Pediatría 2000; 71: 405-12.

19. Medina E, KaEMPFFer AM. Mortalidad del adulto en Chile. Rev Méd Chile 2000; 128: 1144-9.

20. Sánchez H, Albala C, Leva L Años de vida perdidos por muerte prematura en adultos de Santiago, chemos ganado con equidad? Rev Méd Chile 2005; 133: 575-82.
21. Canals M. Patrones estacionales de enfermedades infecciosas: similitud y distancias. Rev Méd Chile 1997; 125: 474-82.

22. Szot J. Mortalidad infantil e indicadores económicos en Chile 1985-1999. Rev Méd Chile 2002; 130: 107-12.

23. Sánchez H, Albala C. Desigualdades en salud: mortalidad del adulto en comunas del Gran Santiago. Rev Méd Chile 2004; 132: 453-60.

24. Donoso E. Desigualdad en mortalidad infantil entre las comunas de Santiago. Rev Méd Chile 2004; 132: 461-6.

25. Gajardo J, Olmos A, Seguel I. Sobrevida de pacientes diabéticos con enfermedad coronaria conocida. Rev Méd Chile 2004; 132, 331-6.

26. Medina E, KaEMPFFer AM. La salud en Chile: reflexiones para el futuro. Rev Méd Chile 1990; 118: $1165-70$. 